\title{
On the Innovative Expression of Contemporary Pastel Art Sheng $\mathrm{Bi}^{1.2}$ and Bao Chen ${ }^{3}$
}

1Jingdezhen Ceramic University, Jingdezhen, Jiangxi, 333000;

${ }^{2}$ China Academy of Fine Arts, Hangzhou, Zhejiang 31000;

${ }^{3}$ College of Science and Technology, Jingdezhen Ceramic University, Jingdezhen, Jiangxi, 333000

\begin{abstract}
Jingdezhen's porcelain industry reached its peak in the Qing Dynasty, during which pastel was formed due to some specific social, cultural, political and economic reasons. This article mainly focuses on the artistic characteristics of its ceramic decorative language, through its expression technique, modeling, subject matter and so on. It mainly discusses the possibility of its artistic language expression. And its causes, influence and artistic value are analyzed and studied.
\end{abstract}

Keywords: Jingdezhen, Pastel decoration, Innovation, Contemporary

\section{试论当代粉彩艺术的创新表达}

\author{
毕胜 ${ }^{1.2}$, 陈宝 ${ }^{3}$
}

(1 景德镇陶瓷大学, 江西, 景德镇, 333000;2 中国美术学院, 浙江, 杭州, 31000

; 3 景德镇陶瓷大学科技艺术学院, 江西, 景德镇, 333000)

摘要: 景德镇的制瓷业在清朝达到了㰾峰, 而粉彩正是在这个瓷业巅峰时期形成的, 自然有其特定社会、文化、政治、 经济等方面的原因, 本文主要是以景德镇粉彩为研究主体, 围绕其陶瓷装饰语言的艺术特性, 通过其表现手法、造型、题材 等方面进行渗透式研究, 着重探讨其艺术语言表达的可能性, 对其产生原因和影响以及艺术价值等进行分析和研究。

关键词: 景德镇; 粉彩装饰; 创新; 当代

中图分类号: J021 文摘标识码: A

\section{引言}

粉彩, 粉彩又叫 “软彩”, 其制作工艺为在烧好的素器釉面上进行彩绘, 再入窑经 $600^{\circ} \mathrm{C}$ 至 $900^{\circ} \mathrm{C}$ 温 度烧制而成。粉彩瓷的彩绘方法一般是, 先在高温烧成的白瓷上勾画出图案的轮廓, 然后用含砷的玻璃白 打底, 再将颜料施于这层玻璃白之上, 用干净笔轻轻地将颜色依深浅浓淡的不同需要洗开, 使花瓣和人物 衣服有浓淡明暗之感。粉彩作为陶瓷釉上彩的一种比较成熟的技法, 是经过了宋、元、明、清四个朝代在 工艺、技巧及艺术观念的积累和沉淀, 并吸取了各姐妹艺术中的营养, 采取了点染与套色的手法, 使所要 描绘的对象，无论人物，山水，花卉，鸟虫都呈现出质感强烈，明暗清晰，层次分明的艺术特征。

随着人们价值观念的多元化, 景德镇当代粉彩艺术也与时俱进, 不断创新, 以满足人们审美观念、审 美价值、审美情趣与审美追求多元化的需要。景德镇当代粉彩艺术创新的内在动力在于审美价值观的多元 化。十一届三中全会以后进入了陶瓷艺术创作的春天, 百花齐放春满园, 瓷苑出现了一片繁荣景象。陶瓷 艺术家在绘画题材, 绘制工艺, 表现手法都有新的发展。在表现手法上, 既有重工粉彩, 也有写意人物, 既有边饰与主题人物相祄托的民族艺术风格，也有以主题画面装饰，不用边饰的国画手法，“密不通风”、 “疏可走马” 的不同布局，古代、现代人物形象，应有尽有，雅俗共赏，工笔、意笔乃至抽象风格的人物 形象，个有特色，显示出陶瓷艺术家的不同个性。 


\section{1 艺术内容的创新}

创新, 顾名思义就是指抛弃旧的, 建立新的。创新是一个古老而宽泛的概念, 起源于拉丁语, 早在 15 世 纪, 人们就开始使用创新一词, 其原意是引入新东西、新概念。这是宇宙间普遍存在的新陈代谢客观规律 的一种反映。一般意义上讲的创新, 是从积极因素考虑的, 兼顾到行为的时效性、实用性、价值性等因素。 一项伟大的发明和创新可能对整个社会文明的进步带来巨大的推动作用。反之，一些不能够达到上述结果 的创新, 有可能是无效的或者说是多余的。所以, 我们说, 创新也应分为有效的或有价值的创新和无效的 或无价值的标新立异。在自然创新层面, 创新的社会意义不能绝对值化; 在艺术创新层面, 创新的效果也 能绝对值化, 换言之, 不是所有的 “创新” 都能带来好的、正面的效果。因此, 具体到粉彩艺术创新领域, 也存在有效的、有价值的创新和无效的无意义的创新。要想强化这一点, 就得从每一个艺术创作者自身出 发, 寻找到有价值的有意义的艺术创新。艺术的创新源于对艺术具有较深的领悟, 通过对传统的、原有的 事物进行合理地传承和有价值的改造，因此，艺术的创新不是无水之源、无根之木，而是有一定的依凭。

景德镇粉彩艺术的内容创新, 在于从事粉彩创作的艺人对陶瓷绘画的独特理解和切身实践, 将陶瓷绘 画的表现功能充分运用到粉彩创作中去, 钟莲生教授提出 “在当今的陶瓷艺术领域, 已形成了陶瓷装饰、 陶瓷绘画、现代陶艺三足鼎立的局面，但人们对这三种艺术形式的概念、概念的内涵和外延以及他们之间 的交叉关系, 还并不十分清楚。这不但影响了人们对这三种陶瓷艺术的认识, 也影响到它们的发展”。近 年来, 人们热衷于对古典传说题材进行创作, 上世纪 80 年代景德镇艺术瓷厂粉彩艺人以四大名著之一的 《红楼梦》中大观园为题材而创作的瓷壁画, 长达 10 米, 画中楼台亭阁掩映于花木山石之间, 假山倒映 在水池中, 各种各样的石头都有着最自然的 “睡姿”, 于花木、人物融为一体, 勾起人们对梦里红楼的追 忆与畅想。[16] 现代粉彩艺人以竹林七贤、桃园结义、精忠报国、才子佳人、仙道神佛等故事传说进行艺 术创作, 在艺术创作中弘扬中国传统文化, 也使得作品更具艺术感染力。再如, 21 世纪初, 由中国工艺美 术大师张松茂创作的粉彩雪景瓷板画《三顾茅庐》图（1），整个画面意境开阔、场面宏大而视觉中心突 出, 场景既符合历史真实意义又调动了多种文化元素的寓意, 在极其微细处彰显作者艺术深厚的艺术功底, 在人物刻画方面, 极其注重人物神情, 求贤若渴是这件作品要重点表达的主题。在视觉效果上, 在黑与白 的苍茫间透露出极具绚丽色彩, 给人一种恢弘而不失细淢的审美享受。

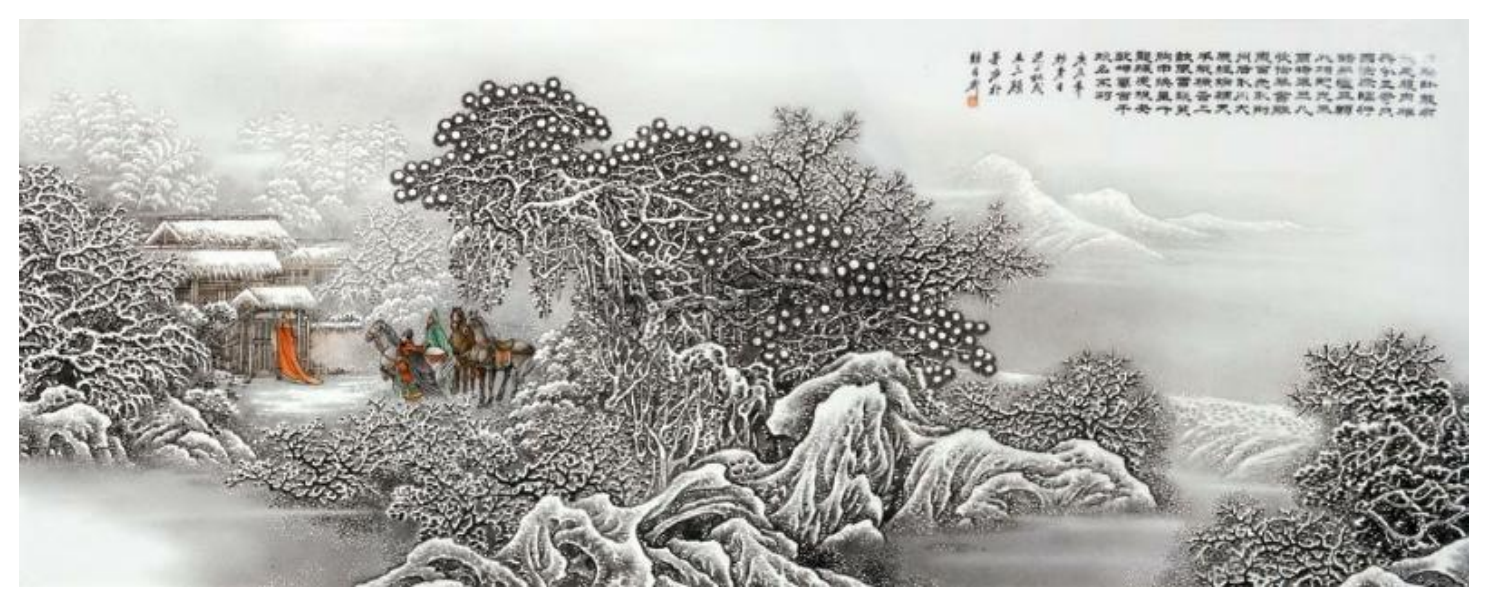

图 (1) 三顾茅庐 张松茂 


\section{2 艺术表现形式的创新}

在形成景德镇艺术创作浓厚的氛围中，许多国内外人士做出了功不可没的贡献。他们不仅给景德镇粉 彩艺术创新带来新的灵感和新的艺术思想, 甚至在提升粉彩艺术审美价值方面也起到了很大的作用。邹晓 松教授认为, 现代陶艺的概念是将装饰、造型, 甚至包括纹理统一结合在一起的, 只不过中国的陶艺类别 可能更多的强调装饰效果，因为在中国传统艺术当中很多都是以色、线、形等元素来表述的，跟西方艺术 在形式上等有很大的差异, 这种特征同时也大量出现在其他艺术设计领域中, 如服装、染织、建筑等, 中 国传统陶瓷的装饰蕴含着对传统文化的一种崇尚, 或者说传统装饰表达某一些美好的意念的时候更注重、 更有效的运用的是装饰语言。陶瓷装饰要博采众长, 形式多样。特别是新时期艺术家对传统粉彩艺术的发 展经过反思与探讨, 普遍认为熔诗书画印于一炉的艺术形式并不是惟一的粉彩式样。粉彩既可认为是陶瓷 器物上的装饰画, 也可作为纯绘画性艺术, 与中国画、油画相比, 只不过是艺术媒质不同而已。认识的变 化促进了新的发展, 这个时期粉彩艺术的主要特征是艺术家各自发挥自己的优势与特长。同时, 又不断挖 掘, 使用新材质, 改进工具, 变换手法和工艺程序等, 创造出新的艺术天地, 这一点在他的作品《荷塘秋 色》（图 2）中表现地淋漓尽致, 作品采用综合装饰的手法, 采用粉彩与颜色釉的相结合的装饰技法, 产 生出不同材料的质地美和形式构造美。

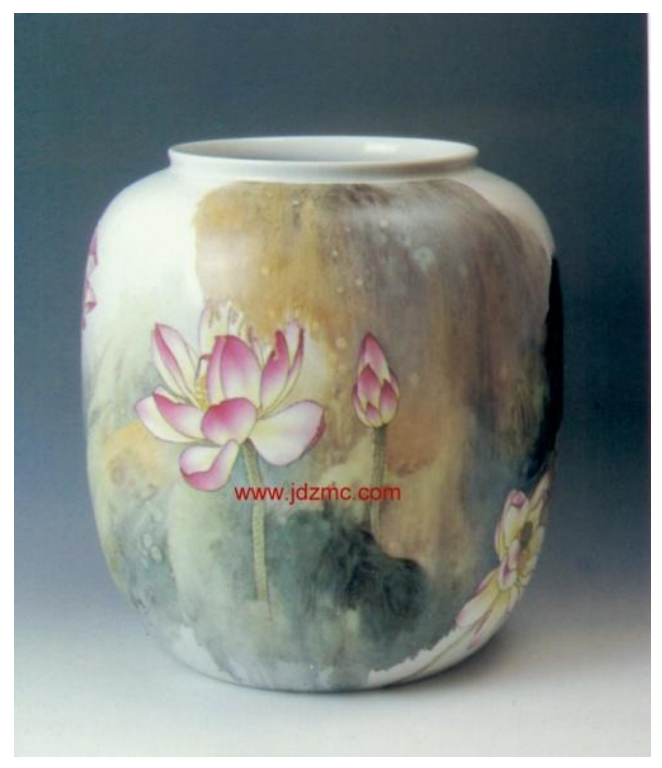

（图 2）《荷塘秋色》

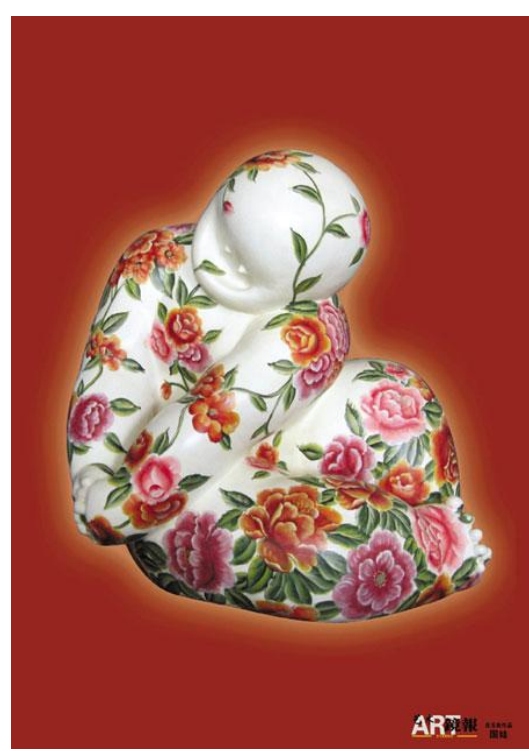

《瓷娃》（图 3）

现代社会, 生活内容更丰富, 人们对美的要求也是千变万化, 粉彩艺人更要把握人们的审美心理, 设 计出具有现代气息, 迎合各种各样的审美要求的新作品。比如粉彩在艺术表现形式上就是极力地追求实用 与审美的最佳结合点。如关玉良创作的《瓷娃》（图 3）系列, 采用当代粉彩技法与雕塑造型结合, 将粉 彩绘画之美与造型之美完美融合，作品中抽象的面孔是为了突出整体，整体就是关玉良想要刻意强调的一 一那就是歌颂生命的力量、阳光一般自由的力量, 瓷娃的特点是光头、胖乎乎和身上充满民族风情的艳丽 花朵图案, 透出一种原始、野性、雄健、蓬勃的生命力。粉彩的艺术创新在于极力地寻求新的适合的表现 方式, 其中, 在造型艺术方面还有很大的开拓创新的空间。当代景德镇粉彩艺术如江河入海, 尽管在先辈 们的呕心沥血下已经成就斐然, 大有汪洋恣意之势, 可作为一门艺术而言, 却还有更多的浩渺的烟波等待 着艺人们去领略, 艺术之海可谓无穷。 
进入新世纪以来, 以学院派画家和青年陶艺家为代表的陶瓷艺术创作群体对现代粉彩的发展有一定的 推动作用。这些新生的艺术群体更加注重当代景德镇粉彩装饰艺术的创新, 如注重粉彩与其他装饰形式的 结合而形成的综合装饰, 从而使装饰内容更为新颖, 风格更为多样化, 融入更多的现代艺术理念, 强烈的 表现了艺术家的思想内涵和艺术境界。如粉彩与其他装饰形式结合而产生的综合装饰：与青花釉里红等釉 下彩的综合装饰，表现出粉润清雅的审美特征；与高温颜色釉的综合装饰，产生秀美浑厚的审美效果；与 新彩等釉上彩的综合装饰，成就俊雅瑰丽、珠联璧合的组合效果。

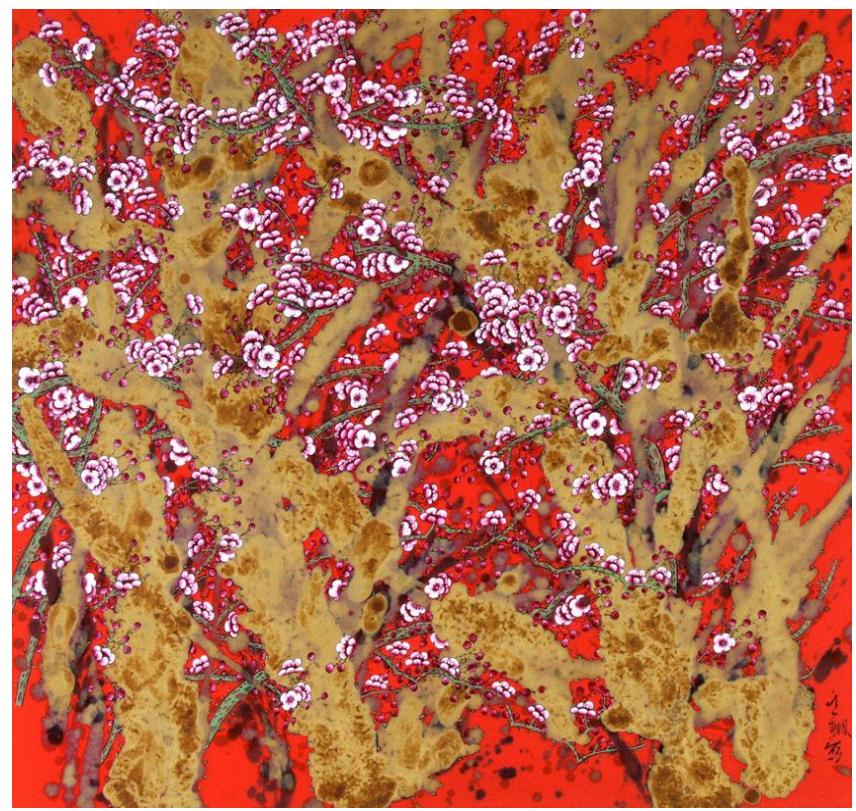

图 4 《腊梅》宁钢

如陶瓷学院教授宁钢老师的作品《腊梅》（如图 4）充分运用颜色釉与粉彩结合进行表现手法上的创 新, 整个画面呈现了寒冬腊月梅花竞相绽放的场景, 采用粉彩绘出梅花的妖娆艳丽, 通过窑变和颜色釉的 方法对腊梅枝干进行艺术处理，从而凸显了梅花枝干的苍虬劲骨。另外，对梅花花瓣采用玻璃白打底后， 其上用洋红洗染，使得明暗合理，进一步增强了画面的层次感。

\section{结论}

笔者通过研究认为, 景德镇当代粉彩艺术创新的内在动力在于审美价值观的多元化。笔者从景德镇当 代粉彩创作理念的更新、艺术内容的创新、艺术风格的创新、表现形式的创新等方面阐述景德镇当代粉彩 艺术的创新表现。研究探索出景德镇当代粉彩艺术创新之路: 继续走艺术本土化道路, 对外来优秀文化兼 容并蓄, 形成自己的艺术特色; 推广陶瓷专业化生产模式, 促进行业分工科学合理, 有效提升工作效率; 在艺术内容方面, 在继承传统装饰题材的基础上, 不断创新题材内容, 使之更加接近人们生活, 更加符合 人们的审美需求; 在艺术风格上, 既不能人云亦云, 随波主流, 也不能标新立异, 画蛇添足, 充分理解风 格创新的内涵, 形成自己的艺术风格, 只有体现了自己风格的艺术作品才有长久的生命力; 在艺术表现形 式上, 对于粉彩器物造型的深刻领会, 应是造型与与装饰构图结合的前提, 将艺术表现在最合适的陶瓷造 型上，使得陶瓷粉彩艺术除了意境美、气势美、结构美、笔墨美、色彩美之外，还有更深层次的工艺美、 材质美、造型美，并且这些美相得益彰。 


\section{参考文献：}

[1]耿宝昌. 明清瓷器鉴定 [M]. 紫禁城出版社, 2001, 4

[2]冯先铭.中国陶瓷 [M] . 上海: 上海古籍出版社, 1995 .

[3] 邹晓松. 传统陶瓷粉彩装饰. 武汉: 武汉理工大学出版社 2005.

[4]邹晓松. 论粉彩肇源的工艺基础 $[J]$. 装饰. 2006

[5]陈雨前, 郑乃章, 李兴华. 景德镇陶瓷文化概论 [M]. 南昌: 江西高校出社. 2004. 10.

[6]探析粉彩艺术的创新表现 作者: 陈宝 毕胜 来源: 《现代装饰 - 理论》 2013 年第 03 期

\section{作者简介:}

毕胜 (1977-) 男, 德镇陶瓷大学讲师, 中国美术学院访问学者, 研究方向为中国画与陶瓷绘画

陈宝 (1984-), 女, 景德镇陶瓷大学科技艺术学院讲师, 研究方向为陶瓷绘画

\section{References:}

[1] Geng Baochang. Porcelain identification in Ming and Qing dynasties [M]. Forbidden City Press, 2001,4

[2] Feng Xianming. China's Pottery [M]. Shanghai: Shanghai Ancient Books Publishing House, 1995.

[3] Zou Xiaosong. Traditional ceramic pastel decoration. Wuhan: Wuhan University of Technology Press, 2005.

[4] Zou Xiaosong. On the Technological Basis of Pastel [J]. Decoration. 2006

[5] Chen Yuqian, Zheng Naizhang, Li Xinghua. An Introduction to Jingdezhen Ceramic Culture [M]. Nanchang: Jiangxi University Press. 2004.10. 